\title{
Relativistic Theory of Spin-Polarized X-Ray Photoelectron Diffraction*
}

\author{
Kouta Ito, ${ }^{\dagger}$ Takehisa Konishi, and Takashi Fujikawa \\ Graduate School of Science, Chiba University, Yayoi-cho 1-33, Inage, Chiba 263-8522, Japan
}

(Received 31 October 2008; Accepted 13 January 2009; Published 21 February 2009)

\begin{abstract}
In this work Spin-Polarized X-ray Photoelectron Diffraction (SPXPD) is studied on the basis of multiple scattering theory in the relativistic theoretical framework. This approach is inevitable to study XPD and SPXPD spectra from heavy elements $(Z>50)$, where relativistic effects play some important roles. In the K-edge excitation spin-orbit interaction for photoelectrons is quite important to produce spin-polarization of photoelectrons by use of circularly polarized light, and that this effect has influence on the branching ratio of multiplet splitting in photoemission spectra. In the case of linearly polarized light this phenomenon is expected to be small. We, however, expect that we can see finite contribution in the forbidden direction. In that direction one of important relativistic effects, spin flip scatterings excited by linearly polarized X-ray photons is remarkable not only in heavy atoms but in light atoms. [DOI: 10.1380/ejssnt.2009.115]
\end{abstract}

Keywords: relativistic QED theory; Multiple scattering theory; Multiplet splitting; Spin-polarized X-ray photoelectron diffraction (SPXPD); Linear polarized light; Circularly polarized light; Dipole approximation

\section{INTRODUCTION}

Magnetic ordering at the outermost surface layers can be different from that in the bulk $[1,2]$. Among several tools for the study of surface magnetic structures, spinpolarized X-ray photoelectron diffraction (SPXPD) is one of the promising methods because its pattern directly provides the information about important local surface magnetic structures [3-5]. XPD patterns give the information on geometric structures at surface, while SPXPD patterns give the information on magnetic structures at surface. There are also theoretical researches about SPXPD in the framework of nonrelativistic theory [6-9]. Here SPXPD is studied on the basis of multiple scattering theory in the relativistic framework. This approach is inevitable in studying the XPD patterns from heavy elements [10]. In the K-edge excitation spin-orbit interaction for photoelectrons is quite important to produce spin-polarization of photoelectrons by use of circularly polarized light. In our previous work we calculated the relativistic SPXPD patterns excited by circularly polarized light and we found that the relativistic effect had influence on the branching ratio of multiplet splitting in photoemission spectra excited from heavy elements [11]. As a related application we theoretically study possible methods how to obtain imaging of magnetic atoms [13] by use of XPD and Daimon effect where forward focusing peaks are rotated around by incident circularly polarized light [12].

In the case of linearly polarized light this phenomenon is not expected to be observed. There are relativistic effects even in this case, which have no influence on the branching ratio of multiplet splitting. We, however, expect that we can see finite relativistic contribution to SPXPD in the forbidden direction: This direction is forbidden within electric dipole approximation. In that direction $(\perp \mathbf{E})$ one of important relativistic effects spin flip scatterings excited by linearly polarized X-ray photons is remarkable

\footnotetext{
* This paper was presented at International Symposium on Surface Science and Nanotechnology (ISSS-5), Waseda University, Japan, 9-13 November, 2008.

†Corresponding author: graduate.stereo3.chiba-u.jp@graduate. chiba-u.jp
}

not only excitation from the heavy atoms but from the light atoms.

We calculate and demonstrate these characteristic features in SPXPD by use of circularly and linearly polarized light.

\section{RELATIVISTIC SPIN-POLARIZED XPS THEORY}

A general one-electron relativistic SPXPD theory has been developed by the present authors on the basis of multiple scattering theory [10]. This theory cannot describe the multiplet splittings in the core-hole states which are basically one of many-body effects. We thus have developed a many-body SPXPD theory based on a relativistic quantum electrodynamic (QED) theory [14, 15]. This theoretical framework provides a unified view of SPXPD, and we can calculate SPXPD patterns by circularly polarized light from magnetic materials which give rise to multiplet splittings. A useful formula of the photoelectron current with momentum $\mathbf{p}$, spin $\sigma$ and kinetic energy $\varepsilon_{p}\left(=p^{2} / 2\right)$ excited by $(\mathbf{k} s) \mathrm{X}$-ray photons is given by [14]

$$
\begin{aligned}
& \mathbf{j}_{\mathbf{p} \sigma} \propto \mathbf{p} \sum_{n}\left|S_{n}\right|^{2}\left|M_{1}+M_{2}+M_{3}+\cdots\right|^{2}, \\
& M_{1}(\mathbf{p}, \sigma)_{\mathbf{c}}=\left\langle f_{\mathbf{p} \sigma}^{-}|\triangle| \varphi_{\mathbf{c}}\right\rangle, \\
& M_{2}(\mathbf{p}, \sigma)_{\mathbf{c}}=\left\langle f_{\mathbf{p} \sigma}^{-}\left|\delta T^{\mathbf{r}} g_{11}^{\mathbf{r}} \triangle\right| \varphi_{\mathbf{c}}\right\rangle, \\
& M_{3}(\mathbf{p}, \sigma)_{\mathbf{c}}=\left\langle f_{\mathbf{p} \sigma}^{-}|Q \triangle| \chi_{\mathbf{c}}\right\rangle,
\end{aligned}
$$

where $\left|\varphi_{c}\right\rangle$ and $\left|\chi_{c}\right\rangle$ are the large and the small components of the core function, $\left\langle f_{\mathbf{p} \sigma}^{-}\right|$is the photoelectron function, $\triangle$ is the electron-photon interaction operator, $g_{11}^{r}$ is nonrelativistic retarded one-electron Green's function. $\delta T^{r}$ include spin-orbit interaction which acts on the photoelectron, $S_{n}=\langle n, N-1|b| 0, N\rangle$ is the nonrelativistic intrinsic amplitude, where $b$ is the core electron annihilation operator, and $Q=(\sigma \cdot \mathbf{p}) /(2 c)$. The different core-hole states $|n, N-1\rangle$ are responsible for the multiplet splittings. $M_{1}$ is nonrelativistic term, $M_{2}$ and $M_{3}$ are relativistic correction terms. This formula (1) is the basis for the SPXPD analyses used in the present study. For 
(a)

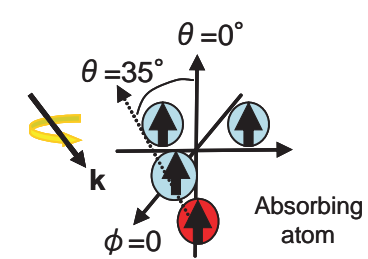

(b)

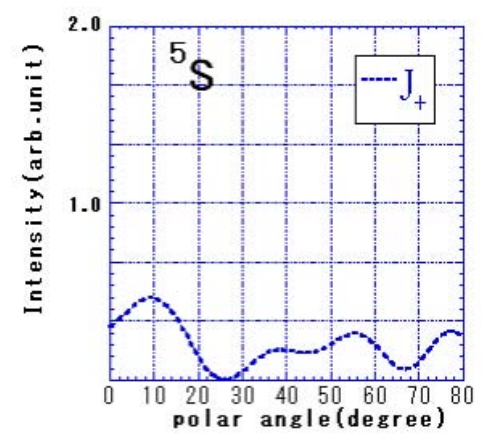

(d)

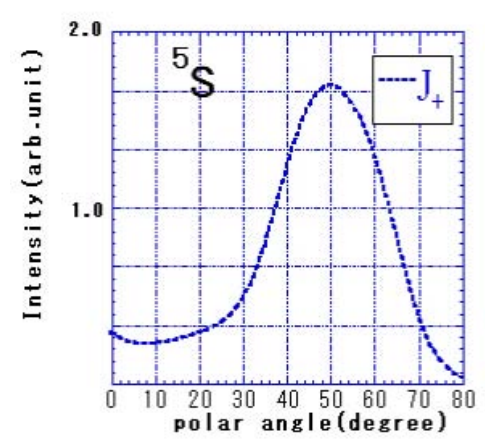

(c)

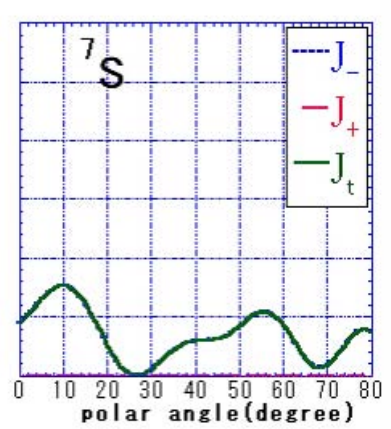

(e)

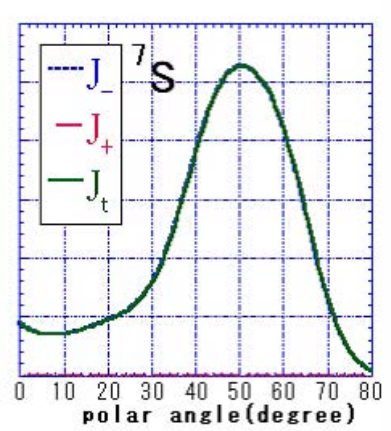

FIG. 1: Artificial model cluster and calculated SPXPD patterns excited from Mn $3 s$ levels for the different final states ( ${ }^{5} S$ and ${ }^{7} S$ ). In (a) three $\mathrm{Mn}$ atoms are in the $\mathrm{x}-\mathrm{y}$ plane and the absorbing atom is on the $\mathrm{z}$-axis in the second layer. We assume fcc $\operatorname{Mn}(111)$ surface in this model. We use $2.52 \AA$ for the Mn-Mn distance. The incident + circularly polarized light propagates in the $\mathrm{x}-\mathrm{z}$ plane $\left(\theta=145^{\circ}, \phi=180^{\circ}\right)$. In (b) and (c) we show the $\theta$ scans ( fixed at $\phi=0^{\circ}$ ) of the SPXPD patterns. In (d) and (e) we show the $\theta$ scans ( fixed at $\phi=180^{\circ}$ ) of the SPXPD patterns. Photoelectron kinetic energy is 100 $\mathrm{eV}\left({ }^{5} S:(\mathrm{b})\right)$ and $104 \mathrm{eV}\left({ }^{7} S:(\mathrm{c})\right)$. The photoelectron inelastic mean free path (IMFP) is set to be $5.0 \AA$. $J_{-}$and $J_{+}$show the intensities measuring down and up spin photoelectrons, and $J_{t}=J_{+}+J_{-}$.

$f_{\mathbf{p} \sigma}^{-}$we can apply site $\mathrm{T}$ matrix expansion, which yields multiple scattering series [16-18].

When the many-body effects in $\delta T^{r}$ are neglected, it is simply written in terms of Hartree potential $V_{H}$ and Pauli spin matrices $\sigma$ as

$$
\begin{aligned}
& \delta T^{r} \sim Q\left(V_{H}-\varepsilon_{p}\right) Q \\
& =-\frac{1}{(2 c)^{2}}\left[\nabla V_{H} \cdot \nabla+\left(V_{H}-\varepsilon\right) \nabla^{2}\right] \\
& \quad+\zeta(r) \sigma \cdot \mathbf{L}
\end{aligned}
$$

where $\zeta(r)$ is related to the spherically symmetric poten-

(a)

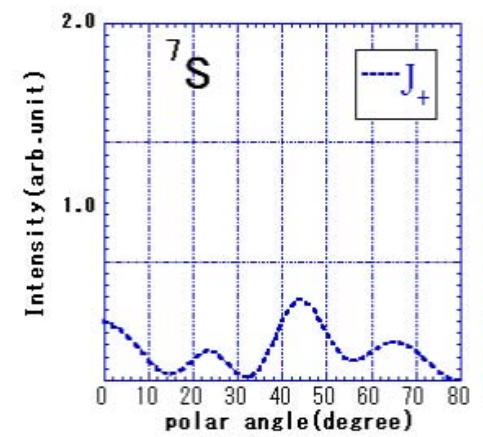

(b)

(c)

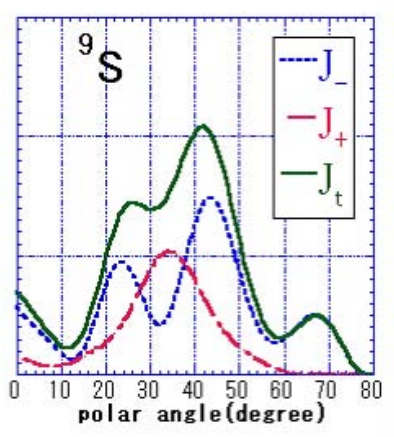

(d)
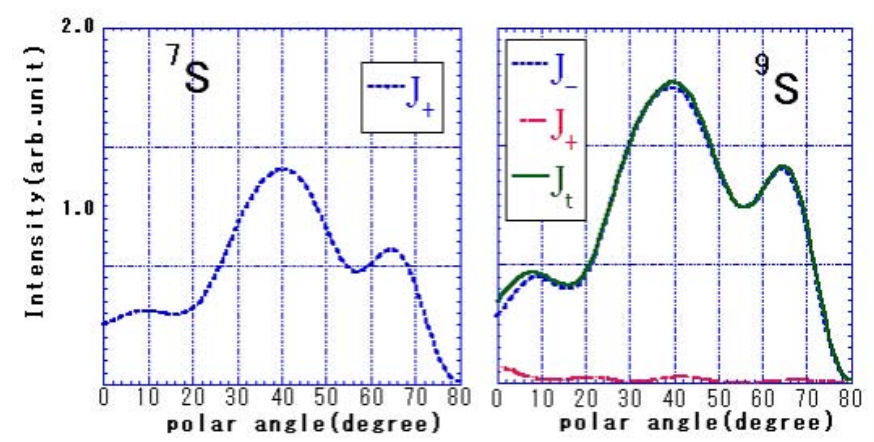

FIG. 2: Gd $4 s \theta$ scan SPXPD patterns for the different final states $\left({ }^{7} S\right.$ and ${ }^{9} S$ ) calculated for the similar model to the one used in Fig.1(a), we assume hcp Gd(0001) surface in this case. We use $3.58 \AA$ for the Gd-Gd distance. In (a) and (b) we show the $\theta$ scans ( fixed at $\phi=0^{\circ}$ ) of the SPXPD patterns. In (c) and (d) we show the $\theta$ scans ( fixed at $\phi=180^{\circ}$ ) of the SPXPD patterns. Photoelectron kinetic energy is $100 \mathrm{eV}\left({ }^{7} S:\right.$ (a),(c)) and $108 \mathrm{eV}\left({ }^{9} S\right.$ : (b),(d)). IMFP is $5.0 \AA$ : $J_{-}$and $J_{+}$show the intensities measuring down and up spin photoelectrons, and $J_{t}=J_{+}+J_{-}$

tial $v_{A}$ at the X-ray absorbing site $\mathrm{A}\left(V_{H}+\Sigma^{a}\left(\varepsilon_{p}\right)=\right.$ $\left.v_{A}+\sum_{\alpha} v_{\alpha}\right)$,

$$
\zeta(r)=\frac{1}{(2 c)^{2} r} \frac{d v_{A}(r)}{d r} .
$$

The operator $\delta T^{r}$ acts on photoelectron wave functions, but the influence is quite small because of the factor $(2 c)^{-2}$. The first two operators in Eq. (2) only change the radial integrals, whereas the third term, the spin-orbit interaction term

$$
\delta V=\zeta(r) \sigma \cdot \mathbf{L}
$$

can contribute to anisotropy in the angular distribution and the spin polarization, therefore it is important in calculating SPXPD patterns from 1s-core levels. We explicitly take that term into account, which yields the approximation for the amplitude $M_{2}$ in Eq. (1)

$$
\begin{aligned}
M_{2} & =\left\langle f_{\mathbf{p} \sigma}^{-}\left|\delta T^{r} g_{11}^{r} \triangle\right| \varphi_{c}\right\rangle \\
& \sim\left\langle\varphi_{\mathbf{p} \sigma}^{0}\left|\left(1+V^{\sigma} G^{\sigma}\right) \delta V G\left(\varepsilon_{p}\right) \triangle\right| \varphi_{c}\right\rangle
\end{aligned}
$$

where $G(\varepsilon)=\left(\varepsilon-T_{e}-V+i \eta\right)^{-1}, T_{e}$ is the kinetic energy operator. $\mathrm{V}$ is the $2 \times 2$ diagonal matrix with $V^{+}$acting 
on up spins and $V^{-}$acting on down spins, and $\varphi_{\mathbf{p} \sigma}^{0}$ is free electron wave function with momentum $\mathbf{p}$ and spin $\sigma$.

In a similar approximation, the amplitude $M_{3}$ in Eq. (1) is given

$$
\begin{aligned}
M_{3} & =\left\langle f_{\mathbf{p} \sigma}^{-}|Q \triangle| \chi_{c}\right\rangle \\
& \sim\left\langle\varphi_{\mathbf{p} \sigma}^{0}\left|\left(1+V^{\sigma} G^{\sigma}\right) \delta U G\left(\varepsilon_{p}\right) \triangle\right| f_{c} y_{j_{c} \mu_{c}}^{l_{c}}\right\rangle
\end{aligned}
$$

where $f_{c}(r)$ is the radial function of the small component $\chi_{c}, y_{j_{c} \mu_{c}}^{l_{c}}$ is Pauli spinor, and $\delta U$ is

$$
\delta U=\frac{1}{2 c r} \sigma \cdot \mathbf{L}
$$

This term can also contribute to anisotropy in the angular distribution and the spin polarization. It is better to estimate the relative importance of $\delta V$ and $\delta U: \delta V$ is in the order of $c^{-2}$ (c=137 in atomic unit), and $\delta U$ is in the order of $c^{-1}$. The former is in $M_{2}$ and the latter is in $M_{3}$ which includes the small component $\chi_{c}$ of the core function in the order of $O\left(\frac{\varphi_{c}}{c}\right)$. Finally we understand that both $M_{2}$ and $M_{3}$ are in the same order.

We can apply the site T-matrix expansion to $G$ in Eqs. (5) and (6), then we get multiple scattering series for the amplitudes $M_{2}$ and $M_{3}$, which are summarized as follows.

$$
\begin{aligned}
& M_{1}(\mathbf{p}, \sigma)_{\mathbf{c}}+M_{2}(\mathbf{p}, \sigma)_{\mathbf{c}}+M_{3}(\mathbf{p}, \sigma)_{\mathbf{c}} \\
& =Z_{1}(\mathbf{p})_{\mathbf{c}}{ }^{\sigma}+S C_{1}(\mathbf{p})_{\mathbf{c}}{ }^{\sigma}\left(M_{2}\right)+S F_{1}(\mathbf{p})_{\mathbf{c}}{ }^{\sigma}\left(M_{2}\right)+S C_{1}(\mathbf{p})_{\mathbf{c}}{ }^{\sigma}\left(M_{3}\right)+S F_{1}(\mathbf{p})_{\mathbf{c}}{ }^{\sigma}\left(M_{3}\right) \\
& +Z_{2}(\mathbf{p})_{\mathbf{c}}{ }^{\sigma}+S C_{2}(\mathbf{p})_{\mathbf{c}}{ }^{\sigma}\left(M_{2}\right)+S F_{2}(\mathbf{p})_{\mathbf{c}}{ }^{\sigma}\left(M_{2}\right)+S C_{2}(\mathbf{p})_{\mathbf{c}}{ }^{\sigma}\left(M_{3}\right)+S F_{2}(\mathbf{p})_{\mathbf{c}}{ }^{\sigma}\left(M_{3}\right) \\
& +Z_{3}(\mathbf{p})_{\mathbf{c}}{ }^{\sigma}+S C_{3}(\mathbf{p})_{\mathbf{c}}{ }^{\sigma}\left(M_{2}\right)+S F_{3}(\mathbf{p})_{\mathbf{c}}{ }^{\sigma}\left(M_{2}\right)+S C_{3}(\mathbf{p})_{\mathbf{c}}{ }^{\sigma}\left(M_{3}\right)+S F_{3}(\mathbf{p})_{\mathbf{c}}{ }^{\sigma}\left(M_{3}\right) \\
& +\cdots \\
& =R Z_{1}(\mathbf{p})_{\mathbf{c}}{ }^{\sigma}+R Z_{2}(\mathbf{p})_{\mathbf{c}}{ }^{\sigma}+R Z_{3}(\mathbf{p})_{\mathbf{c}}{ }^{\sigma}+\cdots
\end{aligned}
$$

where $Z_{1}, Z_{2}$ and $Z_{3}$ are nonrelativistic direct term, single scattering term and double scattering term respectively. $S C_{1}\left(M_{2}\right), S C_{2}\left(M_{2}\right)$ and $S C_{3}\left(M_{2}\right)$ are relativistic spin conserved direct, single scattering and double scattering term in $M_{2} . S F_{1}\left(M_{2}\right), S F_{2}\left(M_{2}\right)$ and $S F_{3}\left(M_{2}\right)$ are relativistic spin flip direct, single scattering and double scattering term in $M_{2}$. Similarly, $S C_{1}\left(M_{3}\right), S C_{2}\left(M_{3}\right)$ and $S C_{3}\left(M_{3}\right)$ are relativistic spin conserved direct, sin- gle scattering and double scattering term in $M_{3}$, and $S F_{1}\left(M_{3}\right), S F_{2}\left(M_{3}\right)$ and $S F_{3}\left(M_{3}\right)$ are relativistic spin flip direct, single scattering and double scattering term in $M_{3}$. These terms are summarized as $R Z_{1}, R Z_{2}$ and $R Z_{3}$ which represent relativistic direct, single scattering and double scattering term respectively. Finally we obtain a compact formula for the photoelectron intensity $\mathbf{j}_{\mathbf{p} \sigma}^{m_{p}}$

$$
\mathbf{j}_{\mathbf{p} \sigma}^{m_{p}} \propto \mathbf{p} \sum_{A} \sum_{\mu_{c}}\left|R Z_{1}(\mathbf{p})_{\mathbf{A} \mu_{\mathbf{c}}} m_{p} \sigma+R Z_{2}(\mathbf{p})_{\mathbf{A} \mu_{\mathbf{c}}}{ }^{m_{p} \sigma}+R Z_{3}(\mathbf{p})_{\mathbf{A} \mu_{\mathbf{c}}} m_{p} \sigma+\cdots\right|^{2}
$$

excited from the sublevel $\mu_{c}$ on site $A$ for the X-ray circular polarization $m_{p}(= \pm 1)$. In the case of K-edge exci- tation photoelectron intensity is simply given by

$$
\begin{aligned}
\mathbf{j}_{\mathbf{p} \sigma}^{m_{p}} & \propto \mathbf{p} \sum_{A} \mid Z_{1}(\mathbf{p})_{\mathbf{A} \mu_{\mathbf{c}}=\frac{\sigma}{2}} m_{p} \sigma S C_{1}(\mathbf{p})_{\mathbf{A} \mu_{\mathbf{c}}=\frac{\sigma}{2}} m_{p} \sigma\left(M_{2}\right)+S C_{1}(\mathbf{p})_{\mathbf{A} \mu_{\mathbf{c}}=\frac{\sigma}{2}} m_{p} \sigma\left(M_{3}\right) \\
& +Z_{2}(\mathbf{p})_{\mathbf{A} \mu_{\mathbf{c}}=\frac{\sigma}{2}} m_{p} \sigma+S C_{2}(\mathbf{p})_{\mathbf{A} \mu_{\mathbf{c}}=\frac{\sigma}{2}} m_{p} \sigma\left(M_{2}\right)+S C_{2}(\mathbf{p})_{\mathbf{A} \mu_{\mathbf{c}}=\frac{\sigma}{2}} m_{p} \sigma\left(M_{3}\right)+\left.\cdots\right|^{2} \\
& +\sum_{A} \mid S F_{1}(\mathbf{p})_{\mathbf{A} \mu_{\mathbf{c}}=\frac{\bar{\sigma}}{2}} m_{p} \sigma\left(M_{2}\right)+S F_{1}(\mathbf{p})_{\mathbf{A} \mu_{\mathbf{c}}=\frac{\bar{\sigma}}{2}} m_{p} \sigma\left(M_{3}\right) \\
& +S F_{2}(\mathbf{p})_{\mathbf{A} \mu_{\mathbf{c}}=\frac{\bar{\sigma}}{2}} m_{p} \sigma\left(M_{2}\right)+S F_{2}(\mathbf{p})_{\mathbf{A} \mu_{\mathbf{c}}=\frac{\bar{\sigma}}{2}} m_{p} \sigma\left(M_{3}\right)+\left.\cdots\right|^{2} \quad(\bar{\sigma}=-\sigma)
\end{aligned}
$$

When we detect up(down) spin photoelectrons, $\sigma$ is equal $\quad+1(-1)$. In magnetic systems where there is the internal 
(a)

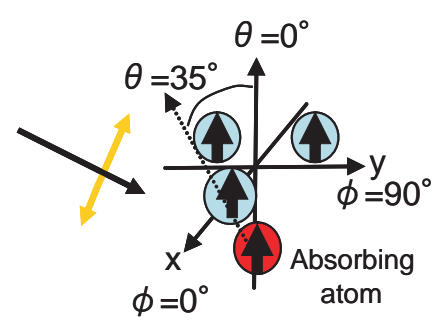

(b)

(c)
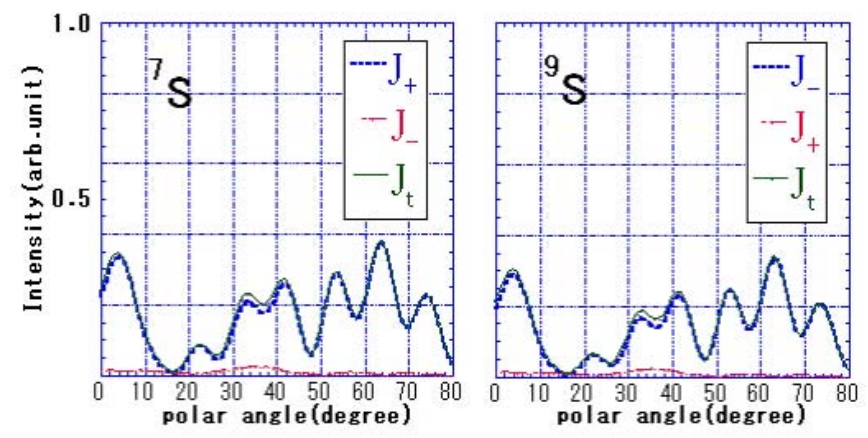

(d)

(e)
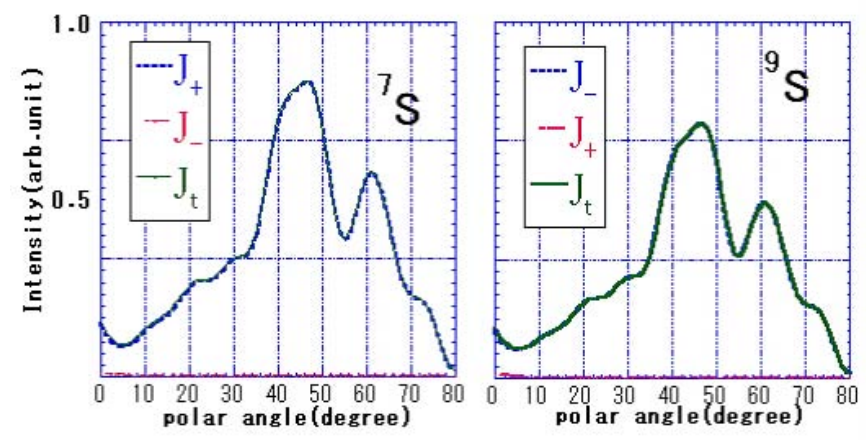

FIG. 3: Artificial model cluster and calculated Gd $4 s$ SPXPD patterns for the different final states $\left({ }^{7} S\right.$ and $\left.{ }^{9} S\right)$. We assume hcp Gd(0001) surface in this case, and use $3.58 \AA$ for the GdGd distance. In (a) three atoms are in the $x-y$ plane and the $\mathrm{X}$-ray absorbing atom is on the z-axis in the second layer. The incident linearly p-polarized light propagates in the $\mathrm{x}$-z plane $\left(\theta=145^{\circ}, \phi=180^{\circ}\right)$. In (b) and (c) we show $\theta$ scan ( fixed at $\phi=0^{\circ}$ ) SPXPD patterns. In (d) and (e) we show $\theta$ scan ( fixed at $\phi=180^{\circ}$ ) SPXPD patterns. Photoelectron kinetic energy is $380 \mathrm{eV}\left({ }^{7} S:\right.$ (b),(d)) and $388 \mathrm{eV} \mathrm{(}{ }^{9} S:$ (c),(e)). The photoelectron inelastic mean free path (IMFP) is set to be $8.5 \AA$. $J_{-}$and $J_{+}$show the intensities measuring down and up spin photoelectrons. $J_{t}=J_{+}+J_{-}$.

magnetic field, there can be multiplet splittings and it is necessary to take into account the splittings of the core levels.

\section{RELATIVISTIC EFFECTS IN SPXPD EXCITED BY CIRCULARLY POLARIZED LIGHT}

In this section we consider Mn 3 s and Gd 4s SPXPD $\left(l_{c}=0\right)$ by use of + circularly polarized light $\left(m_{p}=1\right)$. In this case the spin-orbit coupling only acts on photo- electrons. There is ${ }^{5} S,{ }^{7} S$ final state multiplet splitting spectrum in the photoemission from $\mathrm{Mn} d^{5}\left({ }^{6} S\right.$ ) (high spin state) [3, 4], and ${ }^{7} S,{ }^{9} S$ final state multiplet splitting in the photoemission from $\operatorname{Gd} f^{7}\left({ }^{8} S\right)$ ground state (high spin state) [5].

We first study Mn $3 s$ SPXPD. Figure 1 shows the model used for the present calculations and $\theta$ scan SPXPD patterns for the ${ }^{5} S$ and ${ }^{7} S$ final states. Although Mn crystal has typically a cubic structure, we assume a hexagonal closed-packed (hcp) (0001) or face-centered cubic (fcc) (111) structure to compare the result for Gd crystal (0001) surface with hexagonal structure. We use $2.52 \AA$ for the nearest neighbour Mn-Mn distance, and $3.58 \AA$ for the nearest neighbour Gd-Gd distance.

The higher binding energy peak $\left(\mathrm{Mn}:{ }^{5} S, \mathrm{Gd}:{ }^{7} S\right)$ has only spin-up photoelectrons (spin conserved photoelectrons) and the lower binding energy peak (Mn: ${ }^{7} S$, Gd: $\left.{ }^{9} S\right)$ mainly has spin-down photoelectrons (spin conserved photoelectrons), but it has also spin-up photoelectron (spin flip photoelectrons (the second term in Eq. (10))) by Fano effect $[10,19]$. Specifically, it also contains interference between the relativistic and nonrelativistic spin conserved amplitudes (see the first term in Eq. (10)). We observe nonnegligible differences in the $\theta$ scans for the different final spin states which should be due to exchange scatterings from surrounding magnetic atoms and also due to the relativistic effects.

We can see that the relativistic effects are very small in this Mn $3 s$ SPXPD pattern: $J_{+}$to which only the spin flip process contributes is very small for the ${ }^{7} S$ final state. Figure 2 shows Gd $4 s \theta$ scan SPXPD patterns for the ${ }^{7} S$ and ${ }^{9} S$ final states for the similar model to the one used in Fig. 1, which we assume hcp (0001) structure. In contrast to the case of the Mn $3 s$ we observe a large relativistic effect in the Gd $4 s$ SPXPD pattern. As clearly seen, the relativistic effects are important in calculating the branching ratio $\left({ }^{7} S /{ }^{9} S\right)$ in SPXPD by circularly polarized light from heavy elements like Gd. This is due to the Fano effect: We thus demonstrate that Fano effects strongly affect SPXPD patterns for multiplet splitting final states in heavy elements like Gd.

\section{RELATIVISTIC EFFECTS IN SPXPD EXCITED BY LINEARLY POLARIZED LIGHT}

In case of linearly polarized light or unpolarized light, the relativistic effects could not be very important in SPXPD calculations even for heavy elements, because there is no Fano effect. As we have very small relativistic spin conserved terms, there is quite small interference between the relativistic and nonrelativistic spin conserved amplitudes. We thus expect that the SPXPD patterns are quite similar for the different final spin states: Figures 3(b) and (c) demonstrate the similarity. We thus could think that the relativistic effects play only a minor role in the SPXPD excited by the linearly polarized X-rays. However, when we observe the XPD intensity in the direction where the nonrelativistic photoelectron intensity is very weak, the relativistic effects can be important. This can be understood by the observation that $J_{-}>J_{+}$is satisfied in near normal emission $\left(\theta \sim 0^{\circ}\right)$ in Fig. 4. In this set up, the simple dipole allowed photoemitted electrons 
(a)

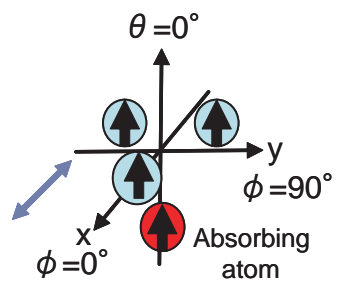

(b)

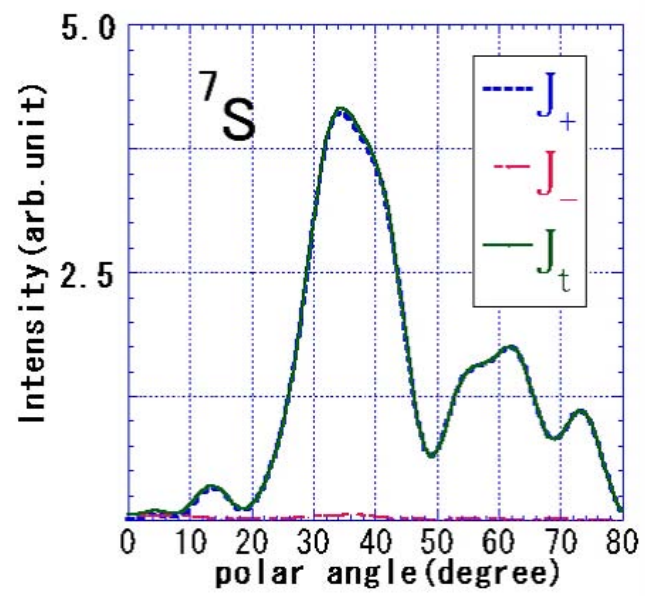

(c)

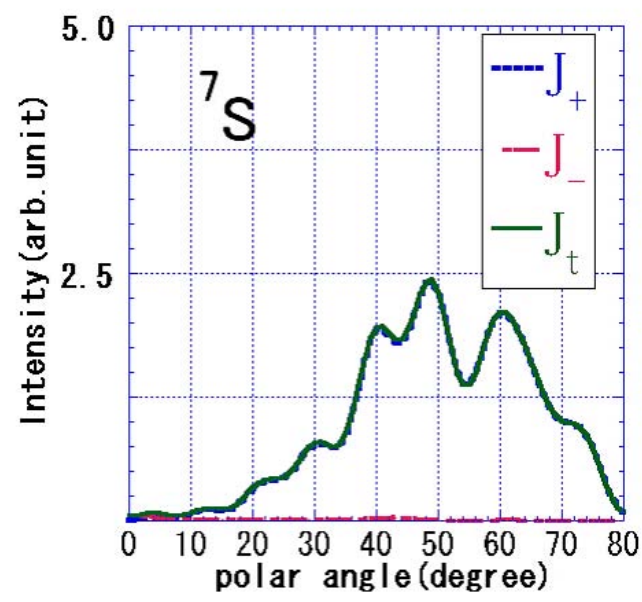

FIG. 4: (a) Artificial model cluster where X-ray is linearly polarized in the x-axis. We assume hcp Gd(0001) surface in this case, and use $3.58 \AA$ for the Gd-Gd distance. (b) Gd $4 s \theta$ scan SPXPD patterns in the $\mathrm{X}-\mathrm{z}$ plane $(x>0)$ for the final state ${ }^{7} S$. (c) $\mathrm{Gd} 4 s \theta$ scan SPXPD patterns in the $\mathrm{x}$ z plane $(x<0)$ for the final state ${ }^{7} S$. Photoelectron kinetic energy is $380 \mathrm{eV}$. IMFP is $8.5 \AA$. $J_{-}$and $J_{+}$show the intensities measuring down and up spin photoelectrons; $J_{t}=J_{+}+J_{-}$

are detected, in particular at $\theta \sim 90^{\circ}$. This selection rule cannot expect photoelectrons at $\theta \sim 0^{\circ}$, the relativistic effects and scattering effects from surrounding atoms can give a finite intensity there. Only the former can contribute to the spin-flip scatterings there. Figure 5 shows that the spin-flip intensity $J_{-}$is much larger than the spin-conserved intensity measuring up spin $J_{+}$, in particular near normal emission. In this set-up we cannot (a)

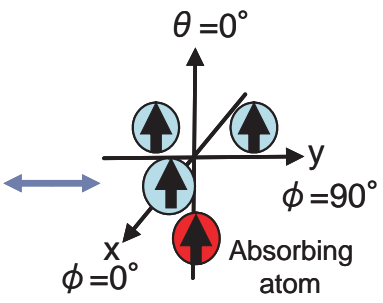

(b)

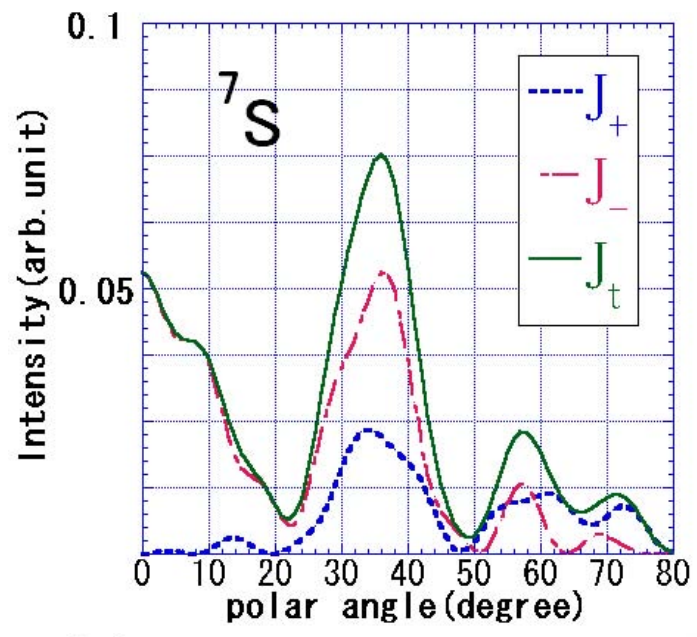

(c)

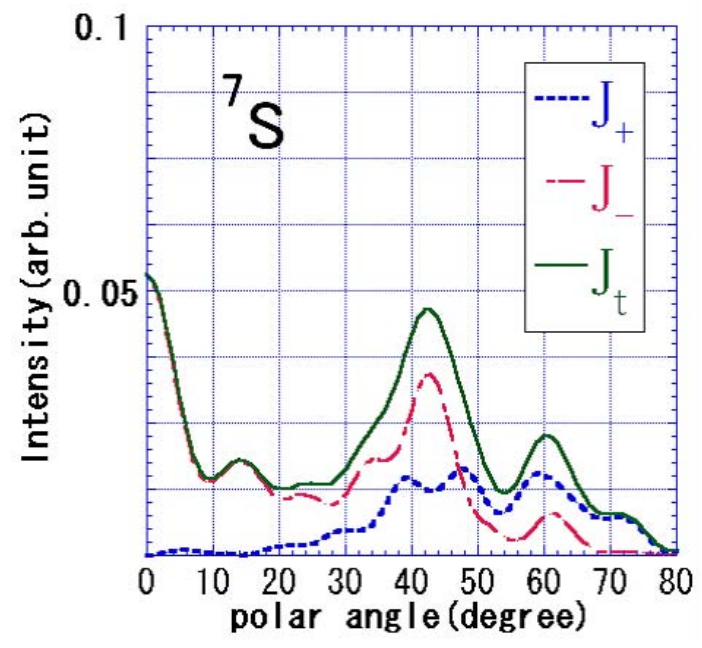

FIG. 5: (a) Artificial model cluster where X-ray is linearly polarized in the y-axis. We assume hcp $\operatorname{Gd}(0001)$ surface in this case, and use $3.58 \AA$ for the Gd-Gd distance. (b) Gd $4 s \theta$ scan SPXPD patterns in the $\mathrm{x}-\mathrm{z}$ plane $(x>0)$ for the final state ${ }^{7} S$. (c) Gd $4 s \theta$ scan SPXPD patterns in the $\mathrm{x}$ z plane $(x<0)$ for the final state ${ }^{7} S$. Photoelectron kinetic energy is $380 \mathrm{eV}$. IMFP is $8.5 \AA$. $J_{-}$and $J_{+}$show the intensities measuring down and up spin photoelectrons; $J_{t}=J_{+}+J_{-}$

detect photoelectrons within nonrelativistic theory, if we neglect elastic scatterings from the surrounding atoms, whereas the relativistic effects and the scattering effects give rise to finite photoemission intensities. That is also the case for Mn $3 s$ photoemission as observed in Fig. 7. Even for light atoms like $\mathrm{Mn}$, the relativistic effects are 
(a)

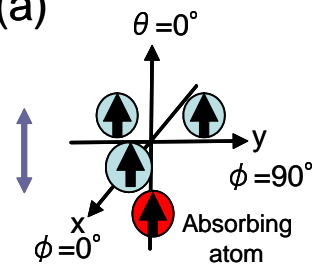

(b)

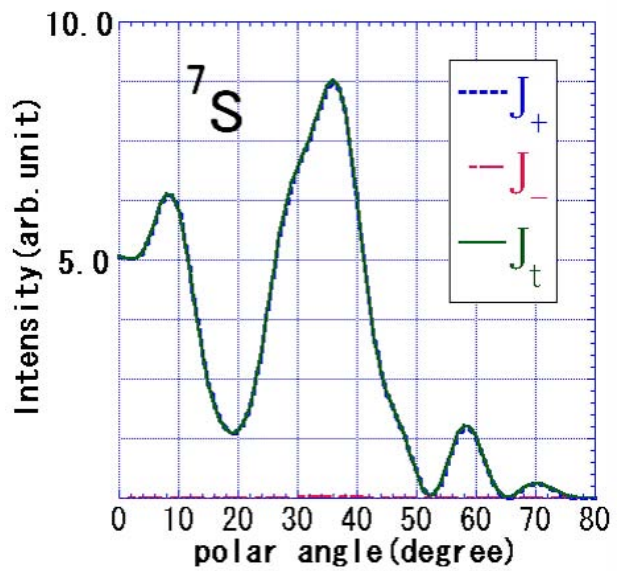

(c)

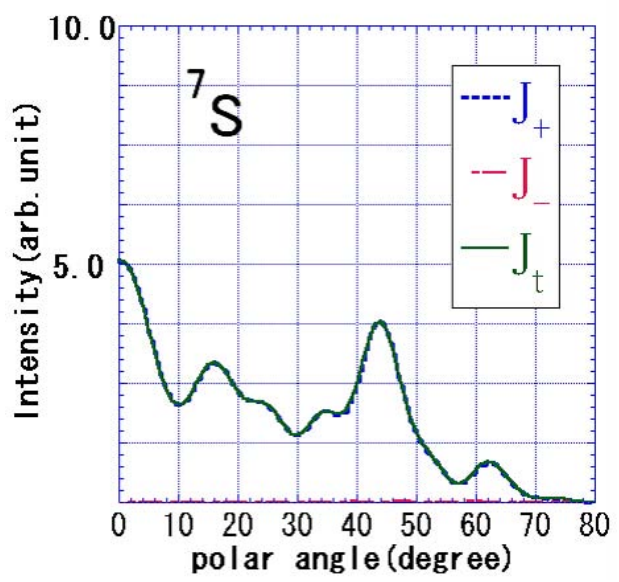

FIG. 6: (a) Artificial model cluster where X-ray is linearly polarized in the z-axis. We assume hcp $\operatorname{Gd}(0001)$ surface in this case, and use $3.58 \AA$ for the Gd-Gd distance. (b) Gd $4 s \theta$ scan SPXPD patterns in the $\mathrm{X}-\mathrm{z}$ plane $(x>0)$ for the final state ${ }^{7} S$. (c) Gd $4 s \theta$ scan SPXPD patterns in the $\mathrm{x}$ z plane $(x<0)$ for the final state ${ }^{7} S$. Photoelectron kinetic energy is $380 \mathrm{eV}$. IMFP is $8.5 \AA$. $J_{-}$and $J_{+}$show the intensities measuring down and up spin photoelectrons; $J_{t}=J_{+}+J_{-}$

sso pronounced, although the absolute intensity is very weak. This is a remarkable feature in SPXPD excited by in-plane linearly polarized (s-polarized) X-rays.

In the case of z-polarized excitation we can say that we cannot observe the relativistic photoelectrons in all direction (Fig. 6). In this case, as the spin flip photoelectrons are mainly emitted to the parallel direction to surface, they hardly can escape from solid surface because of the damping effect. These simulations show that it is essential to consider the relativistic effects when we study XPD excited by in-plane linearly polarized (s-polarized) lights. (a)

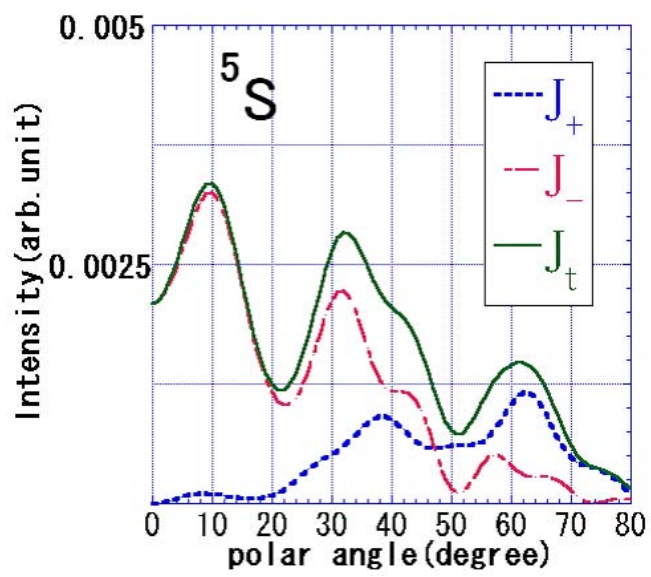

(b)

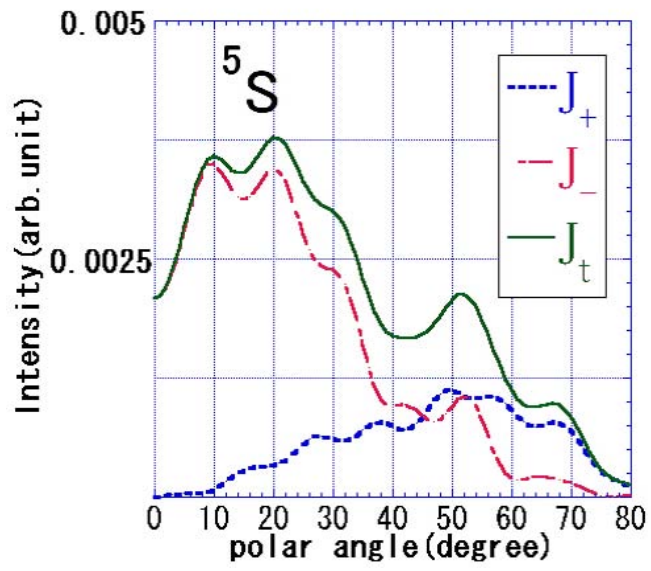

FIG. 7: (a) Mn $3 s \theta$ scan SPXPD patterns in the $\mathrm{x}-\mathrm{z}$ plane $(x>0)$ for the final state ${ }^{5} S$ calculated for the similar model to the one used in Fig.5, we assume fcc $\mathrm{Mn}(111)$ surface in this case. We use $2.52 \AA$ for the Mn-Mn distance. (b) Mn $3 s \theta$ scan SPXPD patterns in the $\mathrm{x}-\mathrm{z}$ plane $(x<0)$ for the final state ${ }^{5} S$ calculated for the same model used in Fig.5. Photoelectron kinetic energy is $300 \mathrm{eV}$. IMFP is $8.0 \AA$. $J_{-}$and $J_{+}$show the intensities measuring down and up spin photoelectrons; $J_{t}=$ $J_{+}+J_{-}$

\section{CONCLUSION}

It is important to include the relativistic terms in the calculations of SPXPD when we use circularly or linearly polarized light as incident X-ray sources. In the case of SPXPD excited by the circularly polarized light the relativistic effects have strong influence on the angular distribution of the intensities of the multiplet-split peaks from heavy atoms: Fano effect is crucial.

In the case of in-plane linearly polarized light the relativistic contribution can became dominant component in the dipole forbidden directions and we cannot neglect it even in the case of light elements. In the case of zpolarized excitation we can neglect relativistic effect because the relativistic photoelectrons almost cannot escape from the solid surface.

In the case of unpolarized light we expect that the relativistic effects play only minor roles, because both Fano 
effect and specific experimental arrangements cannot enhance the relativistic effects. It is thus neccesary to take into account the relativistic effects in the analysis of the complete angular distribution of the photoemission spectra excited by synchrotron radiation.

There are many papers about the MCDAD or MLDAD which use both spin-orbital and exchange splittings [20 $23]$, but they are almost using spin-orbital interaction in the initial state. In this paper we simulate SPXPD patterns which is taken spin-orbital interaction in the photoelectron state into account. We didn't show the MCDAD in this paper, but easily predict that there are large MCDAD in the case of heavy magnetic elements like Gd because there are the nonnegligible spin-orbital interaction in the photoelectron state and enough exchange splitting in the s-core level.
[1] R. Feder (ed.), Polarized Electrons in Surface Physics (World Scientific, Singapore,1985).

[2] C. S. Fadley, Synchrotron Radiation Research, in: R. Z. Bachrach (ed.), Advances in Surface and Interface Science, vol. 1 (Plenum, New York, 1992), p. 421.

[3] B. Sinkovic and C. S. Fadley, Phys. Rev. B 31, 4665 (1985).

[4] B. Sinkovic, B. Hermsmeier, and C. S. Fadley, Phys. Rev. Lett. 55, 1227 (1984).

[5] E. D. Tober, F. J. Palomares, R. X. Ynzunza, R. Denecke, J. Morais, Z. Wang, G. Bino, J.Liesegang, Z. Hussain, and C. S. Fadley, Phys. Rev. Lett. 81, 2360 (1998).

[6] P. Rennert, W. Muck, and A. Chasse, Surf. Sci. 357, 260 (1996).

[7] P. Rennert, J. C. Parlebas, O. Speder, and A. Chasse, Surf. Sci. 331, 1219 (1995).

[8] A. Chasse, W. Kuch, M. Kotsugi, X. Gao, F. Offi, S. Imada, S. Suga H. Daimon, and J. Kirschner, Phys. Rev. B 71, 014444 (2005).

[9] R. Denecke, J. Morais, R. X. Ynzunza, G. H. Fecher, J. G. Menchero, J. Liesegang, J. Kortright, Z. Hussain, and C. S. Fadley, Phys. Rev. B 65, 245421 (2002).

[10] T. Fujikawa and T. Konishi, J. Phys. Soc. Jpn. 72, 2265 (2003).

[11] K. Ito, T. Konishi, and T. Fujikawa, J. Surf. Analysis 14,
328 (2008).

[12] H. Daimon, R. X. Ynzunza, F. J. Palomares, E. D. Tober, Z. X. Wang, A. P. Kaduwela, M. A. Von Hove, and C. S. Fadley, Phys. Rev. B 58, 9662 (1998).

[13] M. Wada, K. Ito, T. Konishi, and T. Fujikawa, Surf. Sci. 602, 2907 (2008)

[14] K. Ito, H. Shinotsuka, T. Konishi, and T. Fujikawa, e-J. Surf. Sci. Nanotech. 3, 353 (2005).

[15] T. Fujikawa, J. Elect. Spect. Relat. Phenom. 136, 85 (2004).

[16] T. Fujikawa, J. Phys. Soc. Jpn. 50, 1321 (1981).

[17] T. Fujikawa, J. Phys. Soc. Jpn. 51, 251 (1982).

[18] T. Fujikawa, J. Phys. Soc. Jpn. 54, 2747 (1985).

[19] U. Fano, Phy. Rev 178, 131 (1969).

[20] R. Denecke, J. Morais, R. X. Ynzunza, J. G. Menchero, J. Liesegang, and C. S. Fadley, J. Electron. Spectrosc. Relat. Phenom. 101, 263 (1999).

[21] A. P. Kaduwela, D. J. Friedman, and C. S. Fadley, J. Electron. Spectrosc. Relat. Phenom. 57, 223 (1991).

[22] M. A. Van Hove, A. P. Kaduwela, H. Xiao, W. Schattke, C. S. Fadley, J. Electron. Spectrosc. Relat. Phenom. 80, 137 (1996).

[23] J. G. Menchero, Phys. Rev. Lett. 76, 3208 (1996). 